\title{
Verdad de paralaje*
}

\author{
Miguel Fonseca**
}

Recepción: 15 de noviembre de 2011 - Aceptación: 18 de enero de 2012

\section{Resumen}

La categoría que se ha querido acuñar como verdad de paralaje (parallax truth), implica la visión de paralaje de los conceptos de verdad ontológica (ontological truth) y verdad epistémica (epistemological truth). Este texto se propone trazar un mapa que permita una ulterior investigación con tal problema como objeto: establecer la brecha de paralaje entre estas dos perspectivas en torno a la verdad.

Palabras clave: Verdad de paralaje, verdad epistémica, verdad ontológica, semántica.

* El presente texto corresponde a las reflexiones surgidas de la tesis de maestría que el profesor Fonseca elaboró en la Universidad Santo Tomás y que pretenden enmarcar el derrotero para el próximo proyecto doctoral.

** El autor es licenciado en filosofía de la Universidad Santo Tomás y Magister en filosofía de la misma institución. Ha trabajado en los seminarios del profesor Spohn en Universität Konstanz y actualmente dirige las cátedras de filosofía analítica y el seminario de Wittgenstein en la Universidad de la Salle. Correo electrónico: miguelfonseca111@gmail.com 


\section{Parallax Truth}

\section{A bstract}

in The category that I want to coin as true parallax involves the parallax view of the concepts of ontological truth and epistemological truth. This paper aims to plot a map that allows for further research with such a problem as the object-matter: to set the parallax gap between these two perspectives regarding the truth.

Keywords: Truth parallax, epistemological truth, ontological truth, semantics. 


\section{Vérité de parallaxe}

\section{Résumé}

La catégorie que j'ai voulu établir comme vérité de parallaxe (parallax truth) implique la vision de parallaxe des concepts de vérité ontologique (ontological truth) et de vérité épistémique (epistemological truth). Ce texte se propose de tracer une carte qui permette une recherche ultérieure avec, pour objet, le problème suivant: établir la brèche de parallaxe entre ces deux perspectives de la vérité.

Mots-clés: Vérité de parallaxe, vérité épistémique, vérité ontologique, sémantique. 
La Verdad de paralaje es una propuesta que se plantea como una mera tentativa para resolver ciertos problemas filosóficos que se generan en la interpretación del concepto de verdad. Las teorías epistémicas y ontológicas sobre la verdad generan brechas insalvables en la hermenéutica del concepto, de tal suerte que solamente podrían ser comprendidos "en una especie de visión de paralaje, que constantemente desplace la perspectiva entre dos puntos para los cuales no hay mediación ni síntesis posible" (Zizek, 2006, p. 11). La única relación entre ellos es quizá, precisamente, la brech a de paralaje, fundamento que permite una ulterior relación entre los ángulos de visión de un mismo fenómeno (Zizek, 2006, p. 12) ${ }^{1}$. La paralaje de realidad y lenguaje, evidente en las esferas ontológicas y epistémicas, puede acuñar una nueva visión de aquello que solemos llamar lo verdadero.

La historia del pensamiento nos ha planteado diversas respuestas al problema de la verdad. Sin embargo, el motivo fundamental que las subsume, desde la misma filosofía griega antigua, hace referencia al carácter funcional de tal concepto; la verdad es una relación. Tal relación funcional es una correspondencia entre cierta idea de mundo y las proposiciones que dan cuenta de él.

Por ejemplo, en la antigüedad se pensaba en la idea de verdad si y solo si tal relación funcional era guiada por el concepto de existencia. La proposición o el estado de cosas al cual se refiere deberían de hecho existir para garantizar la relación y, por supuesto, la verdad. Tal teoría deriva entonces en la clásica visión de la verdad como una correspondencia. Así, la verdad depende de la adecuación de aquello que sea la proposición con los denominados hechos, sean ellos de la índole ontológica que se quiera. Finalmente, podemos hablar de cierta teoría muy contemporánea, la denominada teoría pragmática sobre la verdad que la enuncia como un mero concepto de evaluación, algo similar a un metalenguaje o juego de reglas de la citada relación funcional (Habermas, 1973, pp. 211-265).

1 Mi interpretación de la paralaje, si bien ha sido motivada por las agudas reflexiones de Zizek, más bien está fundamentada en el concepto de paralaje trigonométrica que se usa en la astronomía. Sin embargo. se pueden establecer ciertas relaciones entre este problema y la Paralaje Estelar (primer capítulo de Visión de paralaje) de Zizek que se detiene a analizar la paralaje entre ontología y conocimiento. 
Las teorías anteriormente citadas tienen una tendencia a comprender el concepto de lo verdadero desde las orillas opuestas de lo epistémico y lo ontológico, generando profundas discusiones y horizontes de comprensión de diversos problemas científicos y filosóficos. En este sentido, mi propuesta quiere sumarse a otras (Spohn, 2008) que pretenden identificar estas dos tendencias en torno al problema de la verdad, en una sola visión. Por lo tanto, es preciso realizar un recuento de estos intentos que buscan hermanar las teorías epistémicas y ontológicas de la verdad.

Así, el primer pensamiento que debe ser analizado si se quiere acuñar una verdad de paralaje, es la interpretación del concepto de verdad que realizó Gottlob Frege en sus ensayos sobre semántica, acto fundacional de la filosofía del lenguaje. La teoría sobre el significado de Frege se inscribe en lo que puede ser definido como las teorías que comprenden a la verdad como una correspondencia; la verdad se implica de comprender el significado como una relación entre los símbolos de un lenguaje y ciertas entidades que son independientes de ese lenguaje. Al conjunto de este tipo de teorías se les suele llamar teorías referenciales del significado (Gamut, 2010, pp. 4-5). Como ha sido descrito más arriba, este es un tema con muchas variaciones que dependen de la naturaleza de las entidades a las que los símbolos hacen referencia. Entre ellas, las más destacadas son el platonismo, el conceptualismo y el realismo (Fonseca, 2007).

A pesar de la existencia de tales variantes, la teoría indica que el significado de un símbolo es a lo que éste apunta (Bedeutung) o refiere. Así, la definición que Frege establece en Begriffsschrift (Frege, 1879) es que la verdad es un reflejo de la sintaxis lógica, es decir, de las relaciones funcionales entre el dominio de los símbolos y los referentes. Según Frege los nombres significan objetos, los nombres se convierten en cierto tipo de sustitutos que son usados para reemplazar los objetos a los cuales se refieren. Sin embargo, su carácter de etiquetas o sustitutos no fundamenta totalmente su significatividad; el término no puede ser juzgado como verdadero o falso y, por lo tanto, la palabra no es el núcleo aislado que fundamente la significatividad del signo o nombre. El nombre siempre tiene un modo, una formade significar. El nombre puede ser plenamente significativo en el contexto de la afirmación o sentido (Sinn). Tales expresiones simbólicas 
tienen un correlato ontológico que implica el concepto de verdad como correspondencia. Afirma Gottlob Frege al respecto:

Statements in general, just like equations or inequalities or expressions in analysis, can be imagined to be split up in two parts; one complete in itself, and other in need to supplementation, or'unsaturated' (Frege, 1984, p.146).

El correlato ontológico del significado puede ser entendido en la filosofía de Frege a través de las categorías de objeto y concepto; los objetos y los conceptos mantienen una relación funcional que determina el acaecer de todos los hechos. Una afirmación vincula un objeto con una propiedad y ese es el sentido de la expresión fregeana un objeto cae bajo un concepto. Los conceptos son, en la filosofía de Frege, una especie particular de funciones que mapean objetos verdaderos que caen bajo ciertos límites. El concepto es la estructura, modo, o forma de presentación de los objetos; un concepto es el marco de posibilidad de obtener información sobre los distintos modos de presentación de aquello que entendemos como un objeto (Fonseca, 2009).

Así, una afirmación contiene un sentido, léase correlato del concepto, y un significado o referencia, léase correlato del objeto. Los nombres son "designadores" rígidos de los objetos. Un nombre hace referencia, significa (Bedeutung) a un objeto en el contexto de la forma de la afirmación u oración gramatical (Sinn). Los nombres señalan por lo tanto las partes saturadas de la afirmación

La paralaje fregeana consiste entonces, en primer lugar, en pensar que un referente tiene diferentes modos de hacerse presente al entendimiento. Por ejemplo en una paralaje trigonométrica, un punto $P$ puede ser la intersección de a-b y de a-c. Tal punto medio es la referencia de afirmaciones con diferente sentido, pues nunca es lo mismo el ángulo de visión de a, by c. Por esta razón muchas veces se puede tener claro el sentido de una afirmación, pero no reconocer inmediatamente cuál es el referente $o$, de otro lado, pensar que dos afirmaciones tienen diferentes referentes cuando solamente tienen uno, como en el ejemplo famoso del lucero 
matutino y el lucero vespertino (Frege, 1973). En segundo lugar, la paralaje fregeana parece mostrar que una proposición puede entenderse como verdadera en un plano meramente ontológico, como cuando realizo la paralaje del lucero matutino y el lucero vespertino que de hecho tienen un referente fáctico (Venus); pero también puede hablarse de verdad en un plano meramente epistémico, cuando se suponen los referentes en tanto objetos formales en mundos posibles que no son hechos en el mundo actual, sino que expresan nuestras creencias acerca de cómo son tales hechos en tanto significados cognitivos.

Esta problemática ha sido abordada recientemente por el profesor Wolfgang Spohn, especialmente en su artículo "Two -Dimensional Truth" (Spohn, 2008). En dicho escrito, el profesor Spohn propone que las teorías sobre la verdad, entendidas como ontológicas y epistémicas, tienen la misma relevancia y ninguna subsume a la otra. El carácter bidimensional de la verdad se fundamenta a su vez en una semántica bidimensional. La verdad establece una relación directa con las proposiciones y el mundo o mundos (Armstrong 1997 \& Carpintero 2006). Se habla entonces de una categorización que reconoce truthbearers o proposiciones y truthmakers o mundos. La verdad para el profesor Spohn es una relación entre proposiciones y mundos:

My assumption also entails that truth is basically a relation between proposition and worlds; a proposition is true in a world (and hence true simpliciter, if true in the actual world -whichever that is (Spohn, 2008, p.195).

No obstante, para el profesor Spohn las teorías semánticas que inician con Frege y continúan una tradición en el siglo XX, han nacido con una mácula:

The theories of meaning developed there were burdened with an original sin. Meaning has an ontological aspect, since it compromises reference; with our words we express our believes about what is. (Moreover, we do a lot of things with words; but this is not our present focus) These two aspects were however, hopelessly confused since Frege. The confusion shows up in 
the continuous double purpose intensions and proposition had to, but could not serve, in the continuous indecision between verifiability (or assertibility) and truth conditions, and at many other places (Spohn, 2008, p.198).

Según el profesor Spohn el cambio de perspectiva se da en la obra de Kripke, y su concepto de modalidad (Kripke, 1972). Allí la necesidad y posibilidad epistémica se convierten en asuntos independientes y por lo tanto se consolida la semántica bidimensional que permitiría no hacer jerarquías al hablar de verdad ontológica y epistémica. El asunto que más adelante tratará Putnam consiste en entender que la intensión epistémica requiere de una extensión establecida en tanto probabilidad según una posibilidad ontológica (Putnam, 1975).

In more technical slang, it is the diagonal of the two-dimensional meaning and hence also called diagonal intension. It is clear, thought, that definition of the diagonal involves what I called the Epistemic-ontic map; an epistemic possibility taken as an ontic possibility is just the EO map of the epistemic possibility (Spohn, 2008, p.199).

El EO map, no es otra cosa que comparar las extensiones de dos conjuntos de posibilidades, ontológicas y epistémicas en tanto referentes de las intenciones, en tanto valores de verdad, y establecer sus relaciones según la identidad. Tal concepto es análogo a la brecha de paralaje, en tanto el dominio y codominio de dos conjuntos que pueden coincidir en el rango.

Sin embargo, la gran pregunta que surge es, a saber: ¿qué es una posibilidad epistémica u ontológica? Según el profesor Spohn tendría que ser un mundo, un mundo posible. En este sentido, Spohn afirma que existen dos estilos de mundos, uno lewisiano que definiría las posibilidades epistémicas (Lewis, 1986) y un modelo de mundo wittgensteineano, al estilo del Tractatus que definiría las posibilidades ontológicas (Wittgenstein, 1922). El trabajo consistiría entonces en iniciar un EO map entre proposiciones y mundos lewisianos y proposiciones y mundos al estilo wittgensteineano. 
Tal es la tarea asignada y lo que he decidido llamar el trabajo de construir una verdad de paralaje que dé cuenta de un problema que subsume la mayoría de las estructuras metodológicas de la filosofía y las ciencias. Esta investigación implica entonces determinar las relaciones funcionales entre las proposiciones y los mundos al estilo wittgensteineano y lewisiano, para ulteriormente establecer las posibles relaciones y diferencias entre tales concepciones sobre la verdad, es decir, establecer la brecha de paralaje entre ellas. En este sentido se hace necesario retomar la polémica entre la lógica proposicional estándar y las lógicas modales, a fin de determinar los dominios y relaciones a las cuales cada una de ellas hace referencia.

El presente texto es entonces un primer punto de referencia que requiere de un trabajo más profundo y prolijo. En este sentido podría afirmarse que estamos contemplando nuestra estrella desde el solsticio del verano, esperando pacientemente el invierno que revele la distancia de paralaje que pretendemos.

\section{Referencias bibliográficas}

Armstrong, D. (1997). A World of States of affaires. Cambridge: Cambridge University Press.

Carpintero, M. (2006). The foundations of two dimensional semantics. Oxford: Clarendon Press.

Fonseca, M. (2007). Discurso sobrelos límites del Lenguaje. Bogotá: USTA.

Fonseca, M. (2009).The Quest for the Numbers: An aproach to Frege's philosophy. Revista Logos.

Frege, G. (1879). Begrifftschrifft. Halle: Louis Nebert.

Frege, G. (1984). On Funktion and concept. New York: Basil and Blackwell.

Frege, G. (1973). Sobre Sentido y Referencia. Barcelona: Ariel. 
Gamut. (2010). Lógica, Lenguaje y Significado. Bogotá: Universidad del Rosario.

Habermas, J. (1973). Wahrheittheorien. Pullingen: Neske.

Kripke, S.A. (1972). Name and Necessity. Dordrecht: Reidel.

Kutschera, F. (1979). Filosofía del Lenguaje. Madrid: Gredos.

Lewis. (1986). On the plurality of worlds. Oxford: Blackwell.

Putnam, H. (1975). The meaning of meaning. Cambridge: Cambridge University Press.

Spohn, W. (2008). Two-Dimensional Truth. Studia Philosophica Estonica. Vol .11 94-207.

Wittgenstein, L. (1922). Tractatus logico-philosophicus. London: Routledge.

Zizek, S. (2006). Visión de Paralaje. Buenos Aires: Fondo de Cultura Económica 